\title{
High-power, kilojoule laser interactions with near-critical density plasma
}

L. Willingale, P. M. Nilson, A. G. R. Thomas, S. S. Bulanov, A. Maksimchuk et al.

Citation: Phys. Plasmas 18, 056706 (2011); doi: 10.1063/1.3563438

View online: http://dx.doi.org/10.1063/1.3563438

View Table of Contents: http://pop.aip.org/resource/1/PHPAEN/v18/i5

Published by the AIP Publishing LLC.

\section{Additional information on Phys. Plasmas}

Journal Homepage: http://pop.aip.org/

Journal Information: http://pop.aip.org/about/about_the_journal

Top downloads: http://pop.aip.org/features/most_downloaded

Information for Authors: http://pop.aip.org/authors

\section{ADVERTISEMENT}

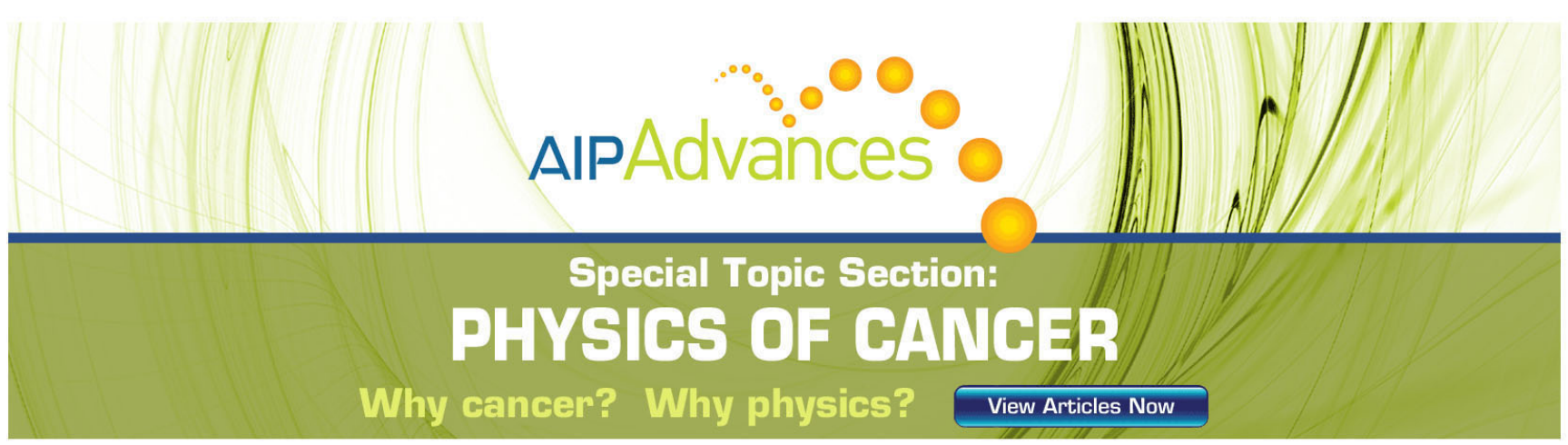




\title{
High-power, kilojoule laser interactions with near-critical density plasma ${ }^{a)}$
}

\author{
L. Willingale, ${ }^{1, b)}$ P. M. Nilson, ${ }^{2}$ A. G. R. Thomas, ${ }^{1}$ S. S. Bulanov, ${ }^{1,3}$ A. Maksimchuk, ${ }^{1}$ \\ W. Nazarov, ${ }^{4}$ T. C. Sangster, ${ }^{2}$ C. Stoeckl, ${ }^{2}$ and K. Krushelnick ${ }^{1}$ \\ ${ }^{1}$ Center for Ultrafast Optical Science, University of Michigan, 2200 Bonisteel Boulevard, Ann Arbor, \\ Michigan 48109, USA \\ ${ }^{2}$ Laboratory for Laser Energetics, University of Rochester, Rochester, New York 14623, USA \\ ${ }^{3}$ University of California, Berkeley, California 94720, USA \\ ${ }^{4}$ University of St. Andrews, Fife, KY16 9ST, United Kingdom
}

(Received 4 December 2010; accepted 14 January 2011; published online 25 April 2011)

\begin{abstract}
Experiments were performed using the Omega EP laser, which provided pulses containing $1 \mathrm{~kJ}$ of energy in 9ps and was used to investigate high-power, relativistic intensity laser interactions with near-critical density plasmas, created from foam targets with densities of $3-100 \mathrm{mg} / \mathrm{cm}^{3}$. The effect of changing the plasma density on both the laser light transmitted through the targets and the proton beam accelerated from the interaction was investigated. Two-dimensional particle-in-cell simulations enabled the interaction dynamics and laser propagation to be studied in detail. The effect of the laser polarization and intensity in the two-dimensional simulations on the channel formation and electron heating are discussed. In this regime, where the plasma density is above the critical density, but below the relativistic critical density, the channel formation speed and therefore length are inversely proportional to the plasma density, which is faster than the hole boring model prediction. A general model is developed to describe the channel length in this regime. (C) 2011 American Institute of Physics. [doi:10.1063/1.3563438]
\end{abstract}

\section{INTRODUCTION}

How relativistic laser pulses interact with near-critical density plasma is of fundamental interest to a broad range of research areas including hot electron production, ${ }^{1}$ proton and ion acceleration, ${ }^{2,3}$ channel formation, ${ }^{4-8}$ high-harmonic generation, ${ }^{2-9} \mathrm{x}$-ray and $\gamma$-ray generation, ${ }^{10}$ and the generation of magnetic fields with strength of hundreds of megagauss. ${ }^{11}$ The critical density, $\mathrm{n}_{\mathrm{c}}$, above which the laser is unable to propagate, occurs when the plasma frequency, $\omega_{p}=\left(n_{e} e^{2} / \epsilon_{0} m_{e}\right)^{1 / 2}$, equals the incoming laser frequency, $\omega_{L}$, so that $n_{c}=m_{e} \varepsilon_{0} \omega_{L}^{2} / e^{2}$. At sufficiently high laser intensities, the electrons oscillate in the fields at speeds approaching the speed of light, $c$. The laser is then able to propagate up to the relativistic critical density, $n_{\gamma c}=\langle\gamma\rangle n_{c}$, where $\langle\gamma\rangle=\sqrt{1+\left\langle a^{2}\right\rangle}$, the normalized vector potential of the laser $a=e E / m_{e} c \omega_{L}$ and $E$ is the electric field of the laser. This is known as relativistically induced transparency. ${ }^{12-16}$

In fast ignition (FI) inertial confinement fusion (ICF), ${ }^{17} \mathrm{a}$ hole boring pulse produces a channel through millimeter scale underdense plasma to access a dense core ${ }^{18}$ and is followed by the ignition pulse to heat the fuel. The expected laser parameters for a channeling pulse to reach the critical density are $\sim 100$ ps at intensities of $I \approx 5 \times 10^{18}-10^{20} \mathrm{~W} \mathrm{~cm}{ }^{-2}$ with an energy of $E_{c} \approx 1.7\left(\mathrm{I} / 10^{18} \mathrm{~W} \mathrm{~cm}^{-2}\right)^{0.36} \mathrm{~kJ} \approx 3-9 \mathrm{~kJ} .{ }^{18}$ Using relativistic transparency may allow the interaction surface to be pushed even closer into the core. ${ }^{7,19,20}$ The ignition energy is usually delivered to the core by a hot electron beam generated at the critical surface, but there are schemes which use ion beams as an alternative ignition source. ${ }^{21,22}$

\footnotetext{
${ }^{\text {a)} P a p e r ~ T I 3 ~ 4, ~ B u l l . ~ A m . ~ P h y s . ~ S o c . ~ 55, ~} 292$ (2010).

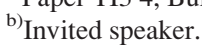

Coupling of the laser energy to the plasma electrons is most efficient near the critical surface $\left(n_{e}=n_{c}\right)$ and all of the phenomena connected with high-intensity laser-plasma interactions are in some way related to how the laser interacts with the electrons. The electron currents generate magnetic fields,${ }^{11}$ which in turn can influence the electron beam transport. ${ }^{23}$ The oscillations of the electrons at the critical surface generate high harmonics. ${ }^{9}$ Ion beams can be accelerated through quasistatic electric fields caused by charge separation (Coulomb explosion, ${ }^{24,25}$ target normal sheath acceleration, ${ }^{26}$ or ponderomotive shock acceleration ${ }^{27}$ ). More efficient ion acceleration can occur at the back of the target through the magnetic field induced longitudinal electric fields. ${ }^{28}$ Propagation of a hot electron beam through a high-Z material can generate positrons through bremsstrahlung. ${ }^{29}$ Therefore it is of great interest, particularly for fast ignition, to understand the intensity scaling for the fraction of the laser energy that couples into the hot electrons, the average hot electron temperature, the angular divergence these electrons acquire, as well as the hot electron beam propagation through plasma.

Creating a near-critical density plasma for wavelengths of around $1 \mu \mathrm{m}\left(n_{c}=1 \times 10^{21} \mathrm{~cm}^{-3}\right)$ with sharp density gradients is experimentally challenging. Solid targets have a very high plasma density once ionized, in the range of $10^{23}$ $10^{24} \mathrm{~cm}^{-3}$. The critical density will be somewhere within the expanding plasma, but there will not be a sharp boundary or region with constant near-critical density over a significant distance. Supersonic gas jet targets are useful for producing plasma densities in the range $10^{18}-10^{20} \mathrm{~cm}^{-3}$, but not much higher. To produce a near-critical density plasma, very low density foam targets are a good choice, as they have a sharp density gradient, with a long uniform initial plasma density 
(determined by the initial target size), which is important for a number of applications. ${ }^{17,28}$

In an overdense plasma, the ponderomotive hole boring model applies momentum conservation to calculate an ion front velocity, ${ }^{1} \quad v_{\mathrm{hb}}=\sqrt{I / m_{i} n_{i} c}=a_{0} c \sqrt{m_{e} n_{c} / 2 m_{i} n_{e}}$, where $a_{0}$ is the peak normalized vector potential. Therefore, taking account of the laser pulse duration an estimate of the laser propagation depth can be made, $d_{\mathrm{hb}} \approx v_{\mathrm{hb}} \tau_{L} \propto$ $\tau_{L} a_{0} / \sqrt{n_{e}}$, where $\tau_{\mathrm{L}}$ is the FWHM laser pulse duration.

Very thin, partially exploded targets ${ }^{30,31}$ or low-density foam targets $^{3}$ have shown improved proton acceleration when the laser is able to propagate all the way through the target. If the laser has propagated through the target, a channel is formed and the fields within the channel act to collimate the hot electron beam, which enhances the electric fields on the rear surface of the target. The resulting enhanced proton acceleration can be used as a diagnostic for laser propagation, and previous results implied that channel formation occurs faster than the hole boring model. ${ }^{3}$ In the regime where $n_{c}<n_{e}<n_{\gamma c}$, the hole boring model does not accurately predict the propagation depth, as the plasma is effectively still underdense to the laser.

This paper presents experimental results and particle-incell (PIC) simulations, which investigate near-critical density interactions. The paper is organized as follows: Section II details the laser system and the low-density foam targets used for the experiments. Plasma density effects are studied by observing the transmitted laser energy and the proton beams accelerated from different density targets. Section III provides details of the PIC simulations that were performed. The effects of polarization, laser intensity, and plasma density on the interaction are investigated with 2D3V PIC simulations and are used to develop a model to describe the length of the channel formed. Finally, the findings are discussed and summarized in Secs. IV and V.

\section{EXPERIMENT}

The experiments were performed using the Omega EP laser system in the Laboratory for Laser Energetics at the University of Rochester. ${ }^{32}$ The central wavelength of the pulse is $\lambda=1.053 \mu \mathrm{m}$ and the beam was linearly polarized. The beam was focused with an $f / 2$ off-axis parabola to a focal spot which was characterized on every shot via phaseretrieval-assisted wavefront measurements ${ }^{33}$ to produce a fluence map. These fluence maps are used to calculate the peak vacuum intensity and peak normalized vector potential, $a_{0}$, for each shot. A typical example is shown in Fig. 1. The main interaction pulse had an on-target energy of $(1000 \pm 11) \mathrm{J}$ with an FWHM pulse length of $(9 \pm 1)$ ps. The peak vacuum intensity for these shots was $(4.2 \pm 1.1) \times 10^{19}$ $\mathrm{W} \mathrm{cm}^{-2}\left(a_{0}=5.8 \pm 0.8\right)$. The laser was focused onto the front surface of the target at normal incidence.

In order that the target has sharp density gradients and near-critical plasma densities, low-density foam targets were fabricated using the in situ polymerization technique. The low-density foams were mounted in washers because of their delicate nature, as described and illustrated in Ref. 3. The foam composition by mass is $71 \% \mathrm{C}, 27 \% \mathrm{O}$, and $2 \% \mathrm{H}$.
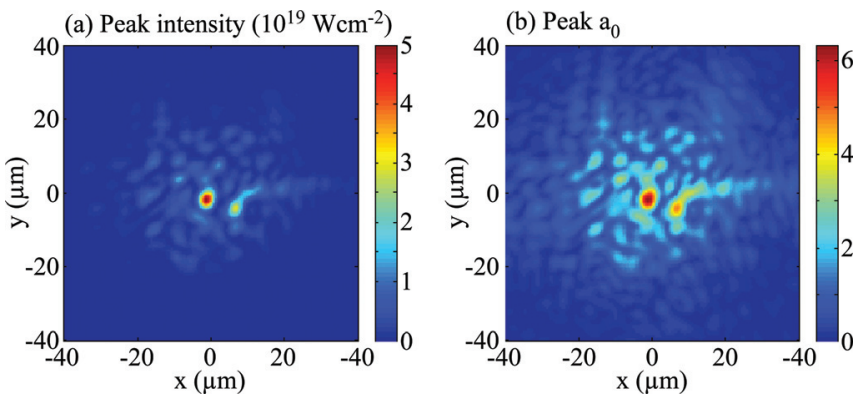

FIG. 1. (Color online) Maps of (a) peak vacuum intensity and (b) peak vacuum normalized vector potential, $\mathrm{a}_{0}$ from a typical 9ps, 996J shot.

Table I shows the maximum plasma electron densities produced from each foam mass density, assuming the laser fully ionized the target. The density structures within the foam are sub micrometer. The interaction pulse had a nanosecond prepulse due to parametric fluorescence, which arrived on the target before the high-intensity short pulse. The intensity contrast ratio between this prepulse and the main pulse was $\sim 10^{-6} .34$ This would be sufficient to preionize the target and homogenize the foam through thermal expansion, but would also create an underdense plasma density ramp on the front side of the target through which the short pulse must travel. Since the target density is low, the critical surface movement is expected to be minimal. The thickness of each foam target was $250 \mu \mathrm{m}$.

A radiochromic film $(\mathrm{RCF})$ stack was positioned in the rear target normal direction (which is also the laser propagation direction) to measure the proton beam. The energy loss for protons peaks near the end of the range of the proton in the material, a feature known as the Bragg peak. This leads to a large signal on the RCF just as the proton was stopped and therefore the signal on each layer was approximately due to a narrow energy spread. With these stacks, the proton beam spectra and divergence were recorded. A diagnostic to monitor the amount of laser light transmitted through the low density targets consisted of a camera imaging the front of the RCF stack. Light transmitted through the target would be scattered from the front surface. A camera imaging the front of the stack was filtered with an interference filter so that only light at $1064 \mathrm{~nm}$ (FWHM transmission of $10 \mathrm{~nm}$ ) was monitored. The transmission of the fundamental laser frequency $1053 \mathrm{~nm}$ through the bandpass filter was $5.5 \%$, and there were additional neutral density filters to further reduce the signal before it was detected by the camera. The

TABLE I. The foam mass densities with the corresponding maximum plasma electron densities assuming a fully ionized plasma $\left(n_{c}=1 \times 10^{21} \mathrm{~cm}^{-2}\right.$ for $\left.\lambda_{L}=1.053 \mu \mathrm{m}\right)$.

\begin{tabular}{lcc}
\hline \hline \multirow{2}{*}{$\begin{array}{l}\text { Foam density } \\
\left(\mathrm{mg} / \mathrm{cm}^{3}\right)\end{array}$} & \multicolumn{2}{c}{ Maximum electron number density } \\
\cline { 2 - 3 } 3 & $\left(\mathrm{~cm}^{-3}\right)$ & $\left(n_{c}\right)$ \\
\hline 10 & $9.0 \times 10^{20}$ & 0.9 \\
20 & $3.0 \times 10^{21}$ & 3 \\
45 & $6.0 \times 10^{21}$ & 6.0 \\
100 & $1.35 \times 10^{22}$ & 13.5 \\
\hline
\end{tabular}


(a) Burn foil

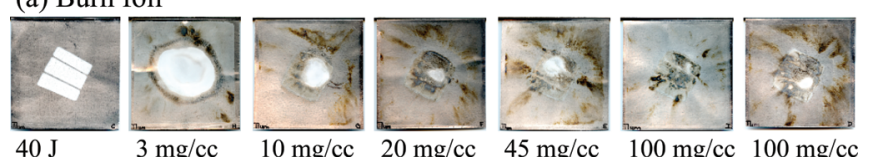

(b) Scattered light images
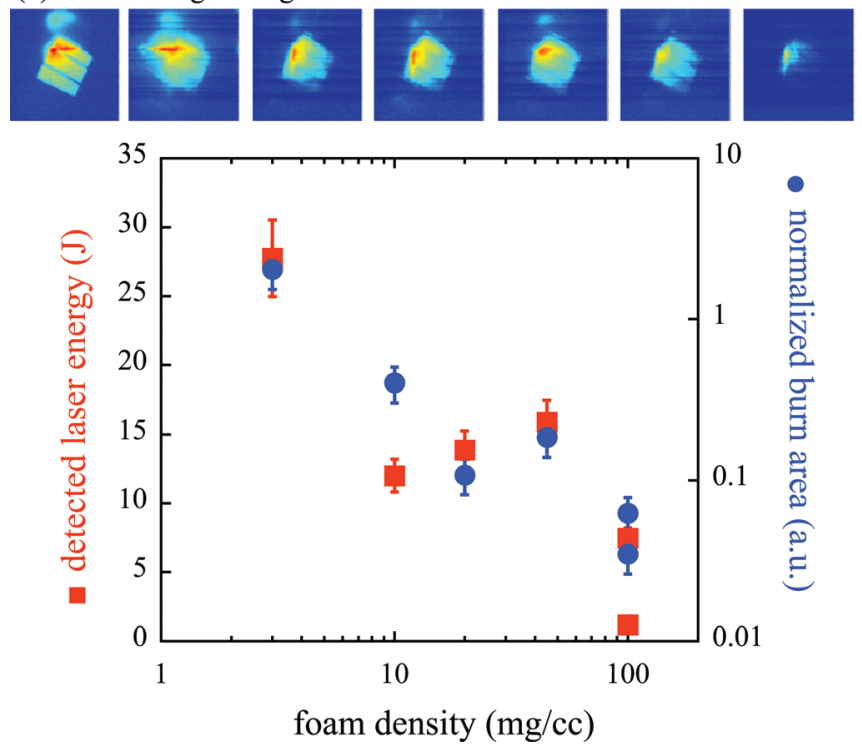

FIG. 2. (Color online) Transmitted laser energy characterization: (a) the burns from the laser damaging the aluminum foil in front of the RCF stack, (b) the images of the transmitted laser light scattered from the front surface, and (c) a plot of the detected laser energy and burn area against the foam density.

front surface of the stack was a flat aluminum foil, which had a roughened front surface so that the scattering was fairly uniform. An energy calibration was performed using a low-energy shot directly onto the front of the RCF stack with no target present.

\section{A. Experimental data}

To characterize the laser energy transmitted through the target both the damage on the front surface of the RCF stack and the scattered light were monitored. The laser damage appeared on the foil as a whitened region, as shown in Fig. 2(a) and this can be characterized in terms of an approximate damage area. The $40 \mathrm{~J}$ calibration shot with no target [left of Figs. 2(a) and 2(b)] shows the square laser profile with the lines corresponding to the regions apodized to prevent diffraction, reflection, and refraction on the tiled gratings. For the target shots, there are other damage features, which appear dark and are likely due to debris from the target. The damage area was reduced with increasing foam density, as shown in Fig. 2. The transmitted laser image [Fig. 2(b)] shows that the total signal decreases with density and suggests only between 0.1 and $3 \%$ of the incident laser energy was transmitted through the foam. However, the $3 \mathrm{mg} / \mathrm{cc}$ shot, where the scattered light diagnostic measures the size of the burn damage was clearly more extensive than the $40 \mathrm{~J}$ calibration shot. There may therefore have been a frequency shift of the laser energy as it propagated through the plasma, moving it out of the transmission window of the bandpass filter. Figure 2(c) plots both the measured laser energy and burn area against foam density.
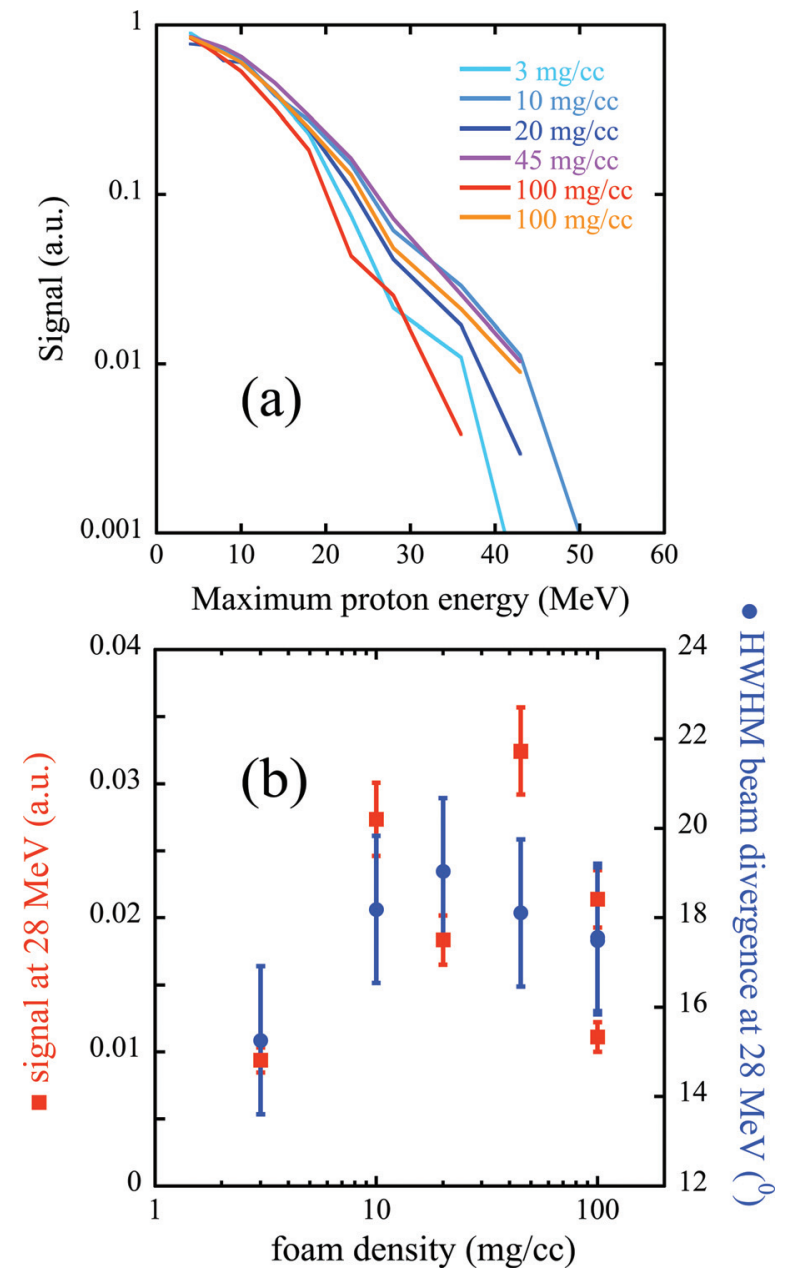

FIG. 3. (Color online) The proton beam data from the RCF stack: (a) proton signal against maximum proton energy for each density target, (b) total proton signal and the HWHM beam divergence at $28 \mathrm{MeV}$ against foam density.

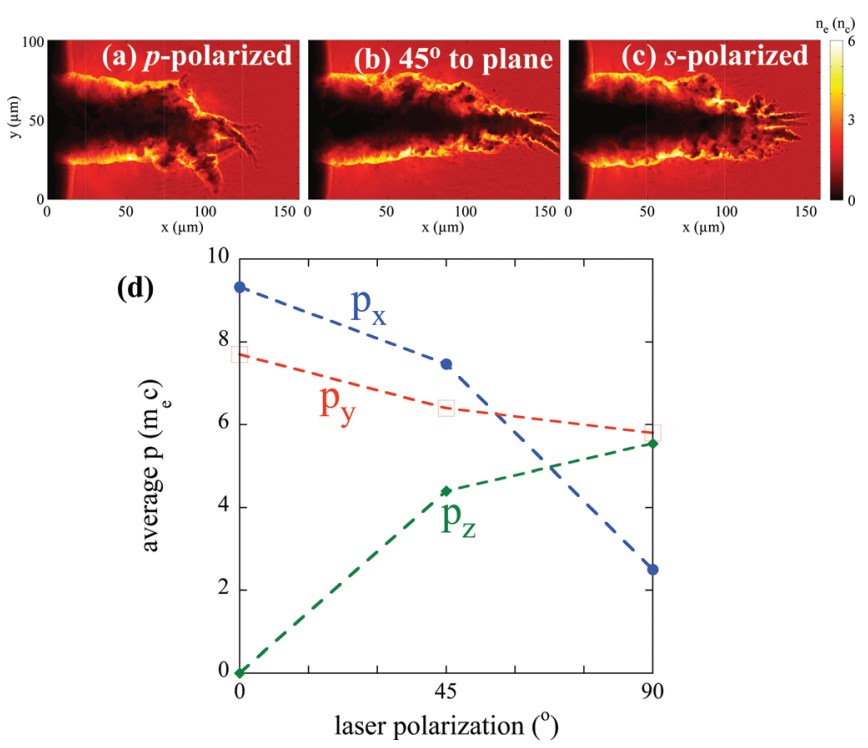

FIG. 4. (Color online) The effect of laser polarization in the 2D simulations on the channel formed in the electron density for the electric field (a) ppolarized (in the plane), (b) polarized at $45^{\circ}$ to the plane, and (c) s-polarized (out of the plane) at a time of $1.7 \mathrm{ps}$ into the simulation. (d) Plot of average $\mathrm{p}_{\mathrm{x}}, \mathrm{p}_{\mathrm{y}}$, and $\mathrm{p}_{\mathrm{z}} \mathrm{vs}$ the angle of the laser electric field to the simulation plane. 

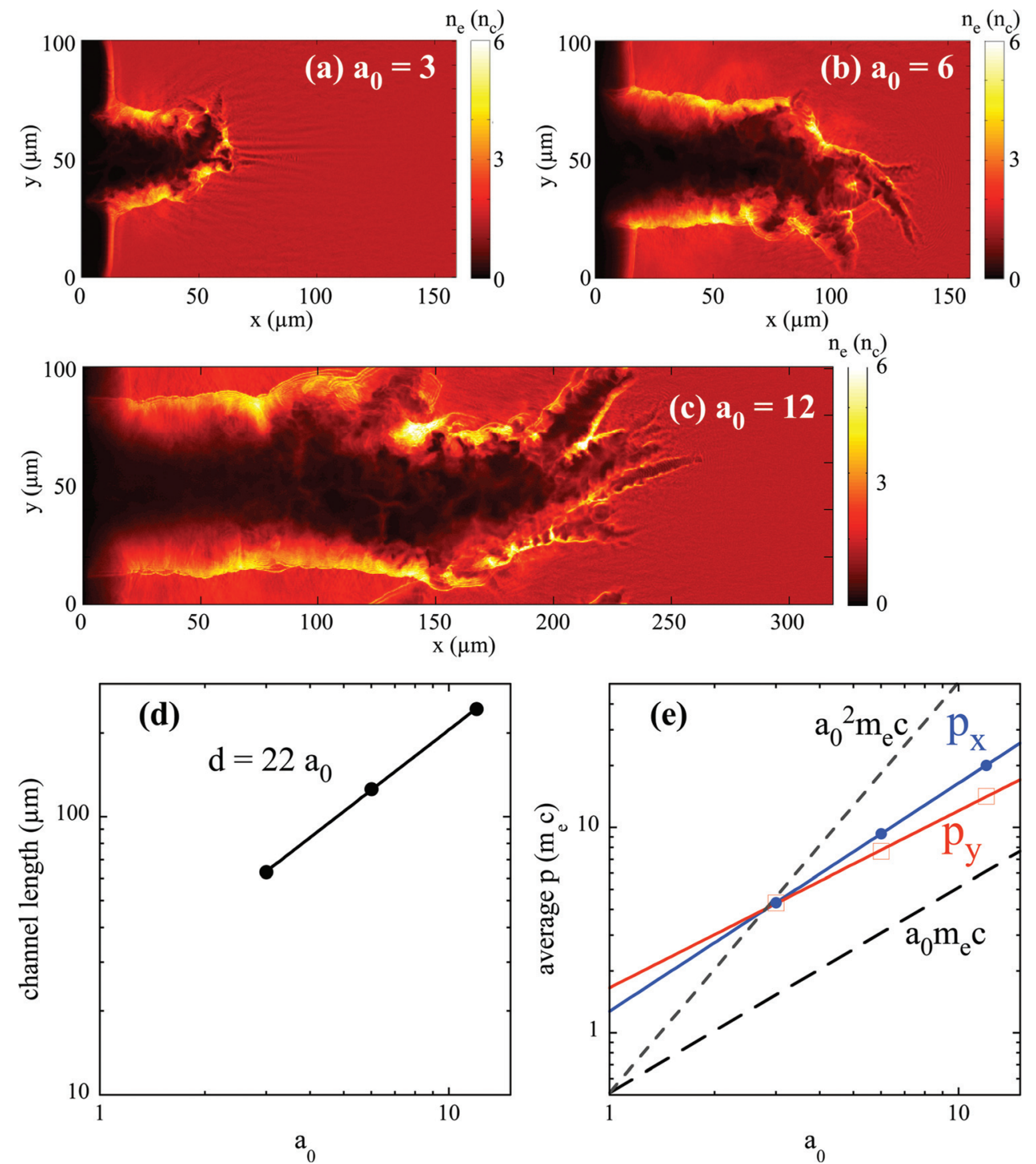

FIG. 5. (Color online) (a)-(c) The final electron densities for each of the intensities simulated and a plot of the final channel length vs $a_{0}$. (d) Plot of the final channel length against $a_{0}$ and (e) a plot of the average $p_{x}$ and $p_{y}$ against $a_{0}$.

The integrated signal on each layer of RCF, which will be dominated by signal from the protons, against maximum proton energy for each layer was plotted in Fig. 3(a) for each of the different shots. At the front of the stack (the lowest proton energy), the RCF signal approaches saturation, making it difficult to compare shots. The maximum proton energy here was not particularly precise as the RCF layer separation was too large to get a high-resolution measurement. Therefore, in order to compare the shots, the total signal on the $28 \mathrm{MeV}$ layer was plotted in Fig. 3(b). There was a general trend of improved proton acceleration as the density increased, but this drops off at the highest density. The best collimated beam was from the $3 \mathrm{mg} / \mathrm{cc}$ target; however the number of protons was lower for this shot and the proton beam also usually becomes more collimated as it approaches the maximum energy.

\section{PARTICLE-IN-CELL SIMULATIONS}

To model the interaction, 2D3V particle-in-cell (PIC) simulations were performed using the osIRIS code. ${ }^{35}$ Parameters which were investigated using the simulations were the polarization of the electric field in the two-dimensional plane, the laser intensity, and the plasma density. The momentum gained by the electrons during these interactions was considered, as well as the laser propagation and consequent channel formation. The energy transfer to the electrons and laser intensity are expected to be important for estimating the expected channel length. The length of the channel was defined for the present analysis as the point furthest through the plasma at which the plasma density had decreased to $75 \%$ of the initial density.

\section{A. Simulation setup}

It would be extremely intensive computationally to model such a large, high-density interaction for the entire 10ps experimental pulse; therefore, a shorter, $1 \mathrm{ps}$ pulse was modeled. The simulation box was defined in the $x-y$ plane, with the laser pulse propagating in the $x$-direction and the $z$-direction being perpendicular to the simulation plane. The laser pulse had a wavelength of $\lambda=1.053 \mu \mathrm{m}$ and was linearly polarized. The temporal profile of the pulse had a rise (and fall) time of $200 \mathrm{fs}$ and flat top of $1 \mathrm{ps}$. It was focused to 

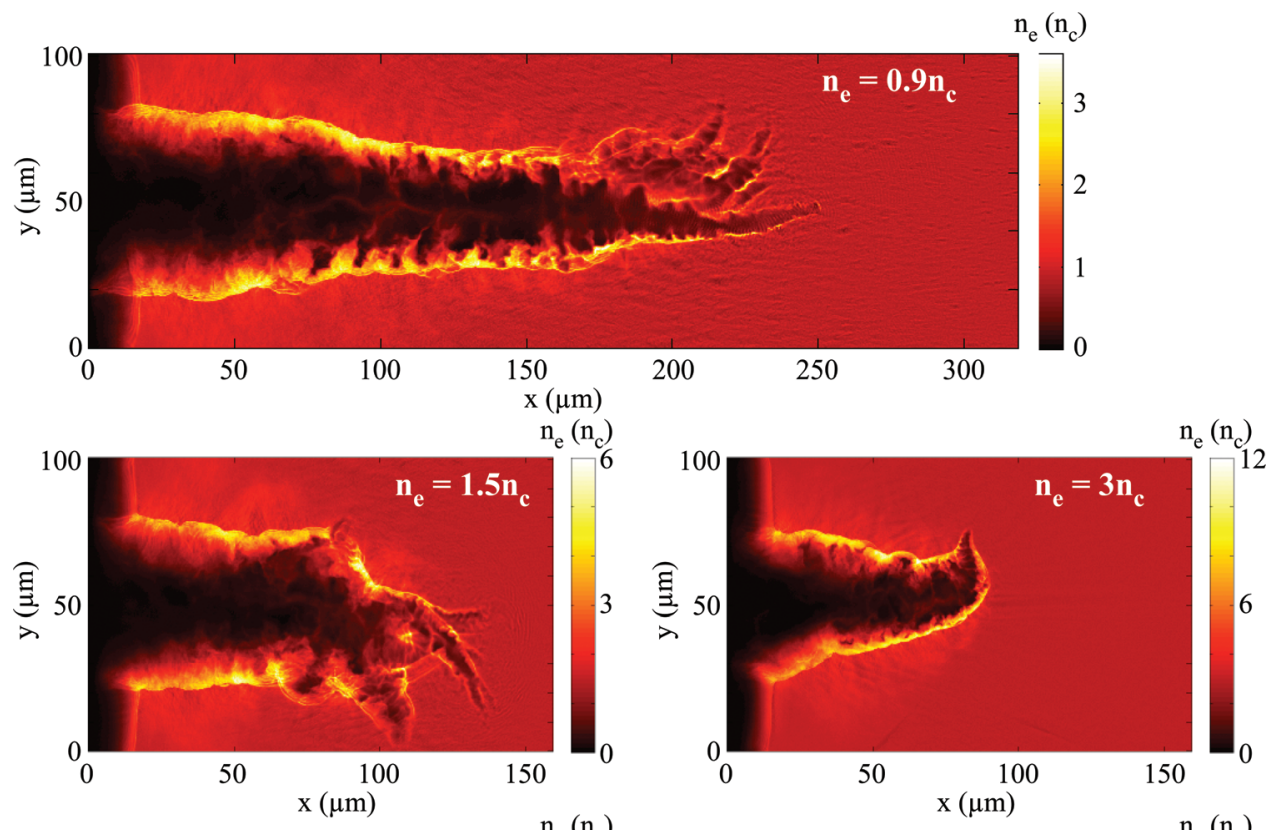

FIG. 6. (Color online) The electron density after the laser fields have dissipated for each of the different initial density simulations.
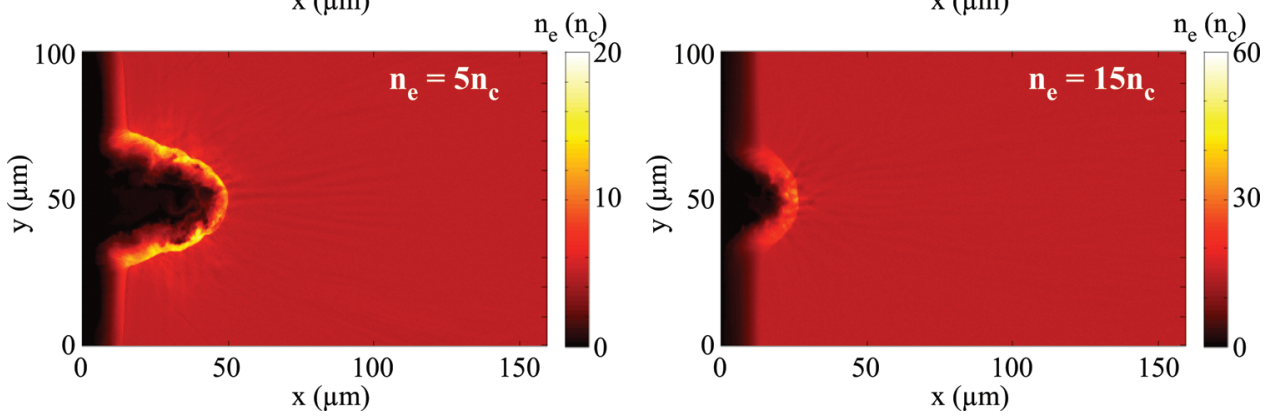

a $1 /$ e radius of $R=11.9 \mu \mathrm{m}$ at the front of the plasma, to give a peak vacuum normalized vector potential, $a_{0}=6$ (unless otherwise stated). The simulation box was $168 \times 100 \mu \mathrm{m}$ with a resolution of 50 cells $/ \lambda$ in the longitudinal $(x)$ direction and 42 cells $/ \lambda$ in the transverse direction $(y)$. There were 12 particles per cell, the ion species used was protons, and the density profile had a $1 \mu \mathrm{m}$ long linear density ramp at the front of the plasma. For simulations where the channel formation went beyond the $168 \mu \mathrm{m}$ longitudinal dimension of the simulation box, the box length was doubled to $336 \mu \mathrm{m}$, but the resolution was maintained.

\section{B. Polarization effects}

The majority of the $2 \mathrm{D}$ simulations presented here are with the electric field of the laser polarized in the plane of the simulation, or $p$-polarized. However, it has been previously recognized that there can be differences in laser propagation through underdense plasma, the speed at which the channel forms, and the spatial development of the channel, if the laser is instead polarized out of the plane ( $s$-polarized). ${ }^{8,18,36}$ Heating of the electrons will also be affected by the polarization, with lower heating expected for $s$-polarized. ${ }^{1}$ Three-dimensional (3D) simulations for the parameters of interest are generally prohibitively computationally intensive, so consideration of different laser polarization geometries in $2 \mathrm{D}$ may provide insight into the experimental interaction. It has been shown that in 3D an anisotropic interaction is expected, perhaps leading to a noncircular channel cross section in reality. ${ }^{37}$ Less laser absorption into the channel walls is expected for $s$-polarized simulations leading to a lower channeling velocity. ${ }^{8}$ For $s$-polarized simulations laser energy can be transfered into slow propagation relativistic solitons and magnetic vortices, whereas magnetic vortex formation is dominant for p-polarization. ${ }^{38-42}$

To identify what differences are likely, simulations with identical parameters, $n_{e}=1.5 n_{c}, a_{0}=6$, but with the electric field polarized either (a) in the plane ( $p$-polarized, $\left.0^{\circ}\right)$, (b) at $45^{\circ}$ to the plane and (c) out of the plane ( $s$-polarized, $\left.90^{\circ}\right)$ were run. The electron densities at a time of $1.7 \mathrm{ps}$ into the simulation are shown for each polarization in Figs. 4(a)4(c). The length of the channel, the channel radius, and the channel formation rate were very similar for all polarizations, but the channel was slightly longer when the electric field is polarized out of the plane. Hosing of the beam was particularly noticeable in the $p$-polarized simulation, with the beam changing direction through large angles; this could be one reason why the channel length was shorter.

The electron momenta were considered at a time of $0.4 \mathrm{ps}$ into the simulation, so that the peak intensity was interacting with the plasma, but boundary effects have not started to influence the spectra. For the different polarization simulations, the average momenta in the $x$-, $y$-, and $z$-directions are shown in Fig. 4(d). The electron heating was most 
efficient when the electric field was in the plane ( $p$-polarized). This was because when the electric field was out of the plane, the electrons could gain momentum in the $z$-direction, but in the 2D geometry they are not moved to a region of lower intensity in the electric field plane over the laser cycle. The lower electron heating for the $s$-polarized simulation may explain the slightly longer channel, as the laser energy was depleted at a slower rate.

\section{Laser intensity}

Keeping the focal spot, polarization ( $p$-polarized), pulse length, and the plasma density constant, $n_{e}=1.5 n_{c}$, the laser energy was varied so that $a_{0}=3,6$, and 12 . Note that the $a_{0}=12$ simulation was performed with a double-length simulation box. Increasing the laser intensity meant the laser propagated further and created a longer channel as presented in Fig. 5 in both the electron densities (a)-(c) and (d) the plot of channel length, $d$, against $a_{0}$. The channel length from these simulations was proportional to $a_{0}$.

The average momentum found in the $x$ - and $y$-directions at a time of $0.4 \mathrm{ps}$ into the simulations, excluding particles with $p_{x}, p_{y}<m_{e} c$, are presented in Fig. 5(e). Also plotted, to illustrate the gradients for these scalings, are $a_{0} m_{e} c$ and $a_{0}^{2} m_{e} c$. The actual scaling for these simulations are $p_{x} \propto a_{0}^{1.1}$ and $p_{y} \propto a_{0}^{0.9}$, which are both close to $p \propto a_{0}$.

\section{Plasma density}

A series of simulation runs were carried out using a laser pulse with $a_{0}=6, p$-polarized, with constant focal spot and pulse length, but the initial plasma density, $n_{e}=0.9 n_{c}$, $1.5 n_{c}, 3 n_{c}, 5 n_{c}, 15 n_{c}$ was varied. The final electron density distributions for each simulation, at a time when the laser fields have dissipated, are shown in Fig. 6 and clearly show that the channels became longer at lower plasma density. Note that the $0.9 n_{c}$ simulation was performed with a double-length simulation box. The length of the channel with time into the simulation was plotted in Fig. 7(a). Plotted in Fig. 7(b) was the total channel length against plasma density and the fit shown was $d[\mu \mathrm{m}] \approx 223 n_{c} / n_{e}$. The channel lengths are much longer than the hole boring estimate suggests, $d_{h b}=\tau_{L} a_{0} c \sqrt{m_{e} n_{c} /\left(2 m_{i} n_{e}\right)}$, but are tending towards $d_{h b}$ at high $n_{e}$.

The average speed at which the leading edge of the channel formation occurs, the channel advancement speed, was calculated for the flat-intensity part of the interaction and is shown in Fig. 7(c). The error bars are given by the standard deviation and the size of these was indicative of the large variation in channel formation speed. As described by Li et al., ${ }^{18}$ the ponderomotive force of the leading edge of the laser pushes plasma ahead, leading to a density increase in front of the laser, which slowed and inhibited propagation. This leads to an intermittent progression of the channel. The slowest speeds were around the hole boring velocity, when the plasma density buildup ahead of the laser had become significant. The group velocity of the laser pulse propagating through the plasma was plotted in Fig. 7(c) and was dependent on the plasma density,
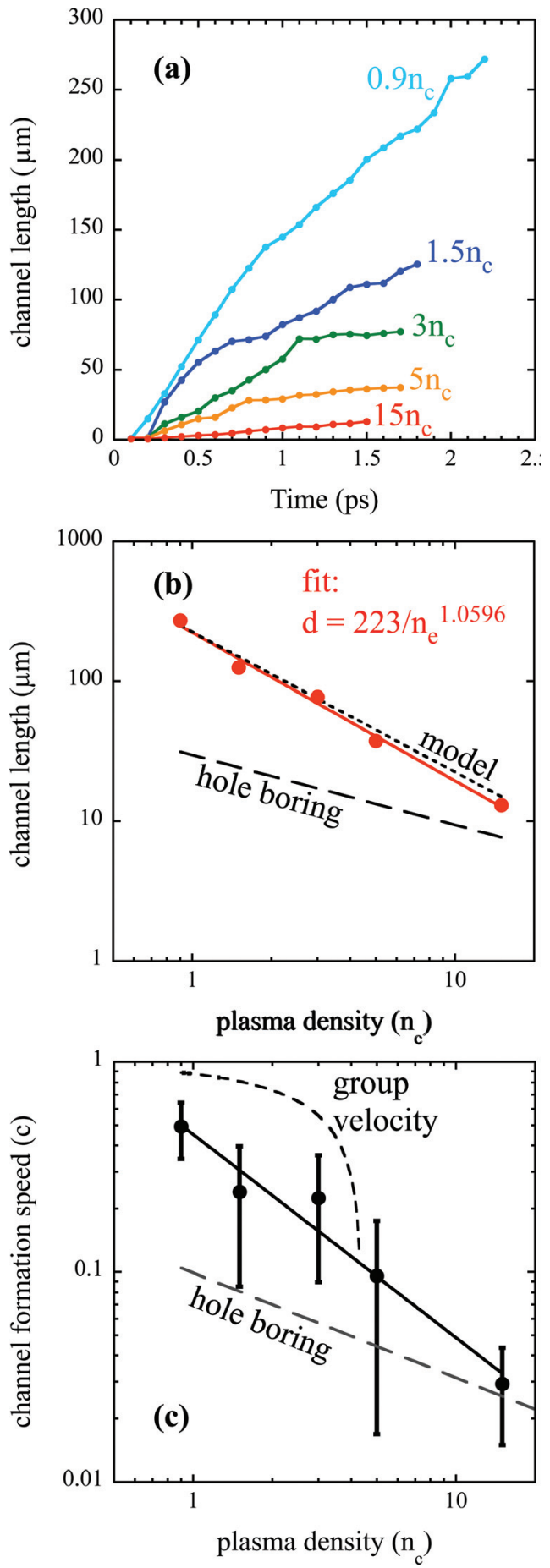

FIG. 7. (Color online) (a) The simulation channel length against time for different plasma electron densities. (b) The channel length once all of the laser fields have dissipated for each of the simulation densities, the fit to the data and the hole boring, and model estimate for channel length as described in Sec. IV. (c) The channel advancement speed during the 1ps time of peak intensity.

$$
v_{g}=\frac{\delta \omega_{L}}{\delta k_{L}}=\eta c=c \sqrt{1-\left(\frac{n_{e}}{\langle\gamma\rangle n_{c}}\right)}
$$

where $\eta$ is the refractive index of the plasma. When $\left.n_{e}\right\rangle\langle\gamma\rangle n_{c}$ the laser is unable to propagate through the plasma.

The transfer of momentum to the electrons also varied with density. The total momentum transferred from the laser 
(a) longitudinal momentum

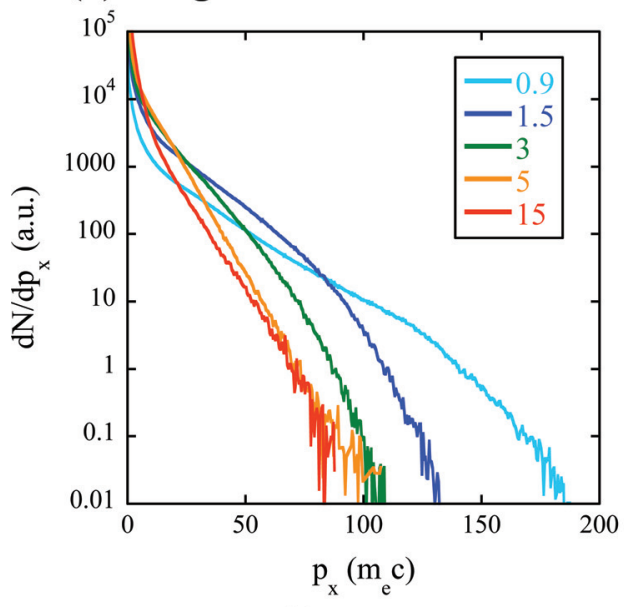

(b) transverse momentum

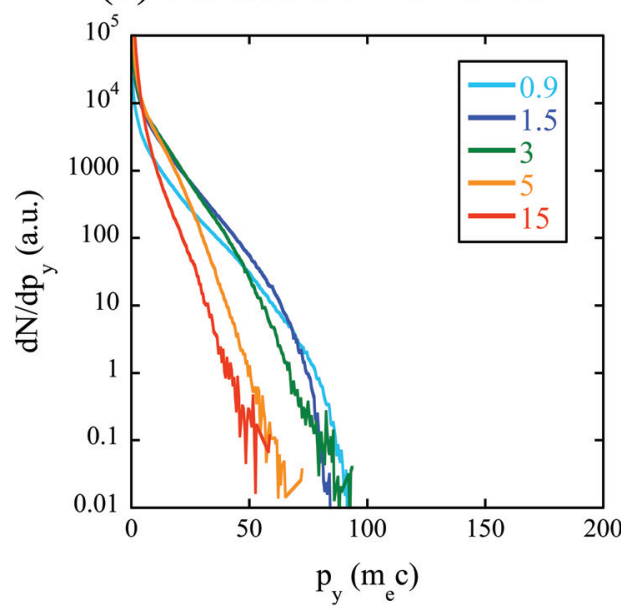

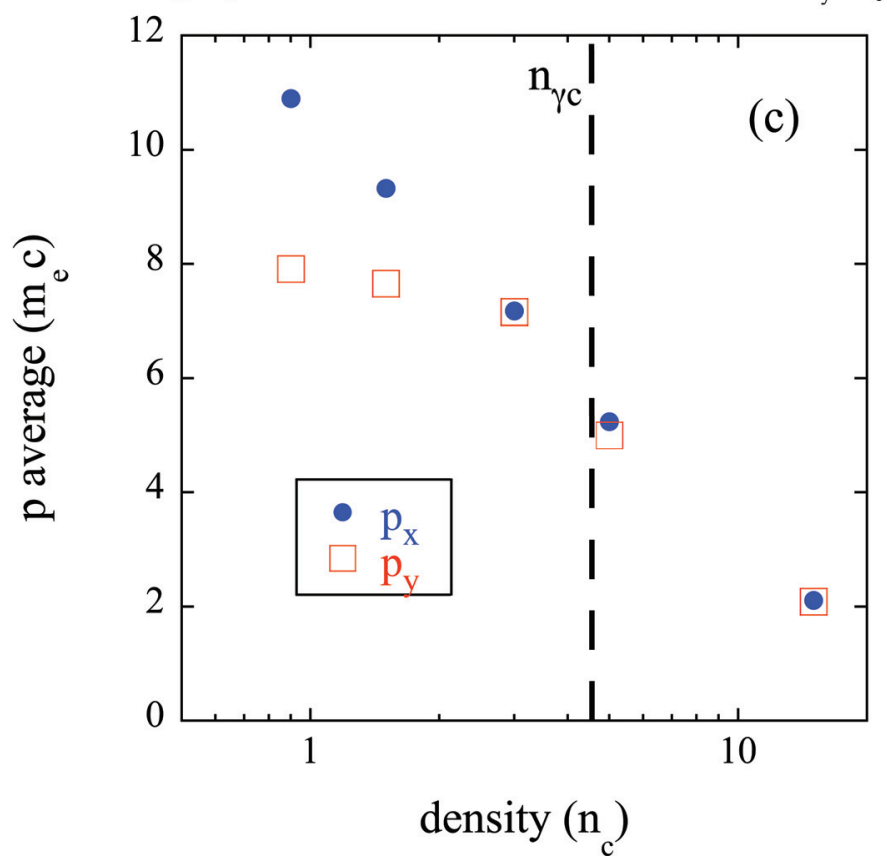

FIG. 8. (Color online) (a) The longitudinal momentum spectra and (b) the transverse momentum spectra for the different density simulations. (c) $p_{\text {fit }}$ to the spectra against plasma density.

fields to the electrons was fairly constant with increasing density. The electron momentum spectra, in both the $x$ - and $y$-directions, at a time of $4 \mathrm{ps}$ into each of the different density simulations, are shown in Figs. 8(a) and 8(b). The longitudinal momentum, $p_{x}$, consistently extends higher than $p_{y}$ for all densities. The average $p_{x}$ and $p_{y}$ found from these momentum spectra, excluding $p_{x}, p_{y}<m_{e} c$ from the calculation, are plotted in Fig. 8(c). Both the $p_{x}$ and $p_{y}$ were higher at the lowest densities, particularly below $n_{\gamma c}$. Also, the longitudinal $p_{x}$ tended to be higher than transverse $p_{y}$ at the lowest densities. It should also be noted that the assessment of the average $p_{x}$ and $p_{y}$ was made at 4 ps to avoid problems with boundary effects, such as particles leaving the box. However, the maximum electron momentum values were often seen at later times, particularly for the lower densities where a significant length channel forms. The electrons could be accelerated via a direct laser acceleration (DLA) mechanism to many times the ponderomotive potential of the laser, $\frac{1}{2} a_{0}^{2} m_{e} c^{2},{ }^{43-48}$ which would lead to a more rapid depletion of the laser energy.

\section{DISCUSSION}

The formation of the channel in the near-critical density plasma was governed by the laser pulse depletion length, i.e., the penetration length, at which all of the laser energy was converted into electrons. ${ }^{3,49}$ The laser pulse energy $\mathcal{E}_{L}=c \tau_{L} \pi R^{2} K m_{e} c^{2} n_{c}\left\langle a^{2}\right\rangle$, where $R$ is the intensity $1 / e^{2}$ focal spot radius and for linear polarization $\left\langle a^{2}\right\rangle=\frac{1}{2} a_{0}^{2}$, was assumed to be equal to the energy transferred to the plasma electrons $\mathcal{E}_{p e}=\bar{\varepsilon}_{e} n_{e} \pi R^{2} d$, where $\mathrm{d}$ is the channel length and $\bar{\varepsilon}_{e}$ is the energy acquired by an electron in the field of the laser pulse. The factor $\mathrm{K}$ arises from the integration over the laser pulse volume and is therefore dependent on the particular temporal and spatial profiles of the laser. Therefore, an estimate of the channel length can be made:

$$
d=K c \tau_{L}\left(\frac{\frac{1}{2} a_{0}^{2} m_{e} c^{2}}{\bar{\varepsilon}_{e}}\right) \frac{n_{c}}{n_{e}} .
$$

Determining the average energy of the electrons, $\overline{\varepsilon_{e}}$, from the simulations in order to prove the assumption we made is 
nontrivial, as the energy transfer mechanism was highly nonlinear. For a long pulse length, a DLA mechanism may have occurred behind the leading edge of the laser pulse in the region where the channel had already formed. The effective temperature of the hot electron tail for DLA is expected to be $\propto a_{0} .{ }^{46}$ A possible issue for fast ignition may be the channeling pulse generating too many hot electrons, which could preheat the core and affect compression. It is also important to understand the speed at which the channel is formed so that the ignition pulse timing can be optimized.

Estimating the average electron energy from the channel to be $\overline{\varepsilon_{e}}=a_{0} m_{e} c^{2}$ means that

$$
d=K c \tau_{L} \frac{a_{0}}{2} \frac{n_{c}}{n_{e}} .
$$

The case of a temporally rectangular pulse, as was used in the simulations here, gave $K=1 / 4$. For $a_{0}=6$ this gave $d[\mu \mathrm{m}] \approx 225 n_{c} / n_{e}$, which was in good agreement with the results of PIC simulations and was plotted in Fig. 7.

For a temporally Gaussian pulse, with an FWHM pulse length of $\tau_{L}$, as used in the experiments, gave $K=0.27$. So, an estimate for the $9 \mathrm{ps}$ pulse used in the experiment has been made, $d=2187 n_{c} / n_{e}$. Therefore, the density at which the channel should break through to the rear side of the $250 \mu \mathrm{m}$ target was $8.8 n_{c}$ (or $\approx 30 \mathrm{mg} / \mathrm{cm}^{-3}$ ). Considering the experimental data, where optimum proton acceleration was seen somewhere between $n_{e}=3 n_{c}$ and $13.5 n_{c}$ provides good agreement with the estimate that the laser breakout at the rear side would occur at $n_{e} \approx 8.8 n_{c}$.

\section{SUMMARY}

In conclusion, a systematic study of high-power, kilojoule laser pulse interactions with near-critical density plasma has been made using experiments with the Omega EP laser facility and particle-in-cell simulations. Very low density foams $\left(3-100 \mathrm{mg} / \mathrm{cm}^{3}\right)$ provided near-critical density targets with an almost constant density profile over relatively long scales (target thickness $250 \mu \mathrm{m}$ ). Transmission of the laser energy was strongly dependent on the foam density. A series of PIC simulations, using a reduced pulse length (1ps) investigated the effects of laser polarization, laser intensity, and plasma density on the electron heating and channel formation. These found the channel length had an $n_{e}^{-1}$ dependence and also was proportional to $a_{0}$.

\section{ACKNOWLEDGMENTS}

The authors gratefully acknowledge the staff of the Laboratory for Laser Energetics for technical assistance during the experiment and the OSIRIS Consortium, consisting of UCLA and IST (Lisbon, Portugal), for the use of the OSIRIS framework. The simulations were run on GlowWorm, part of the Nyx cluster. This work was supported by the National Laser Users' Facility (NLUF) and the DOE (Award No. DEFG52-09NA29041) and NSF grant No. PHY-0935197.

${ }^{1}$ S. C. Wilks, W. L. Kruer, M. Tabak, and A. B. Langdon, Phys. Rev. Lett. 69, 1383 (1992).

${ }^{2}$ E. L. Clark, K. Krushelnick, J. R. Davies, M. Zepf, M. Tatarakis, F. N. Beg, A. Machacek, P. A. Norreys, M. I. K. Santala, I. Watts, and A. E.
Dangor, Phys. Rev. Lett. 84, 670 (2000); A. Maksimchuk, S. Gu, K. Flippo, D. Umstadter, and V. Yu. Bychenkov, ibid 84, 4108 (2000); R. A. Snavely, M. H. Key, S. P. Hatchett, T. E. Cowan, M. Roth, T. W. Phillips, M. A. Stoyer, E. A. Henry, T. C. Sangster, M. S. Singh, S. C. Wilks, A. MacKinnon, A. Offenberger, D. M. Pennington, K. Yasuike, A. B. Langdon, B. F. Lasinski, J. Johnson, M. D. Perry, and E. M. Campbell, ibid 85, 2945 (2000).

${ }^{3}$ L. Willingale, S. R. Nagel, A. G. R. Thomas, C. Bellei, R. J. Clarke, A. E. Dangor, R. Heathcote, M. C. Kaluza, C. Kamperidis, S. Kneip, K. Krushelnick, N. Lopes, S. P. D. Mangles, W. Nazarov, P. M. Nilson, and Z. Najmudin, Phys. Rev. Lett. 102, 125002 (2009).

${ }^{4}$ M. Borghesi, A. J. MacKinnon, L. Barringer, R. Gaillard, L. A. Gizzi, C. Meyer, O. Willi, A. Pukhov, and J. Meyer-ter-Vehn, Phys. Rev. Lett. 78, 879 (1997).

${ }^{5}$ J. Fuchs, G. Malka, J. C Adams, F. Amiranoff, S. D. Baton, N. Blanchot, A. Héron, G. Laval, J. L. Miquel, P. Mora, H. Pépin, and C. Rousseaux, Phys. Rev. Lett. 80, 1658 (1998).

${ }^{6}$ G. S. Sarkisov, V. Yu. Bychenkov, V. N. Novikov, V. T. Tikhonchuk, A. Maksimchuk, S.-Y. Chen, R. Wagner, G. Mourou, and D. Umstadter, Phys. Rev. E 59, 7042 (1999).

${ }^{7}$ A. L. Lei, A. Pukhov, R. Kodama, T. Yabuuchi, K. Adui, K. Endo, R. R. Freeman, H. Habara, Y. Kitagawa, K. Kondo, G. R. Kumar, T. Matsuoka, K. Mima, H. Nagatomo, T. Norimatsu, O. Shorokhov, R. Snavely, X. Q. Yang, J. Zhang, and K. A. Tanaka, Phys. Rev. E 76, 066403 (2007).

${ }^{8}$ N. Naseri, S. G. Bochkarev, and W. Rozmus, Phys. Plasmas 17, 033107 (2010).

${ }^{9}$ B. Dromey, M. Zepf, A. Gopal, K. Lancaster, M. S. Wei, K. Krushelnick, M. Tatarakis, N. Vakaki, S. Moustaizis, R. Kodama, M. Tampo, C. Stoeckl, R. Clarke, H. Habara, D. Neely, S. Karsch, and P. Norreys, Nature Phys. 2, 456 (2006); B. Dromey, S. Kar, C. Bellei, D. C. Carroll, R. J. Clarke, J. S. Green, S. Kneip, K. Markey, S. R. Nagel, P. T. Simpson, L. Willingale, P. McKenna, D. Neely, Z. Najmudin, K. Krushelnick, P. A. Norreys, and M. Zepf, Phys. Rev. Lett. 99, 085001 (2007).

${ }^{10}$ R. D. Edwards, M. A. Sinclair, T. J. Goldsack, K. Krushelnick, F. N. Beg, E. L. Clark, A. E. Dangor, Z. Najmudin, M. Tatarakis, B. Walton, M. Zepf, K. W. D. Ledingham, I. Spencer, P. A. Norreys, R. J. Clarke, R. Kodama, Y. Toyama, and M. Tampo, Appl. Phys. Lett. 80, 2129 (2002).

${ }^{11}$ M. Tatarakis, I. Watts, F. N. Beg, E. L. Clark, A. E. Dangor, A. Gopal, M. G. Haines, P. A. Norreys, U. Wagner, M.-S. Wei, M. Zepf, and K. Krushelnick, Nature, (London) 415, 280 (2002).

${ }^{12}$ P. Kaw and J. Dawson Phys. Fluids 13, 472 (1970).

${ }^{13}$ P. Sprangle, C.-M. Tang, and E. Esarey, IEEE Trans. Plasma Sci. PS-15, 145 (1987).

${ }^{14}$ S. Guérin, P. Mora, J. C. Adam, A. Héron, and G. Laval, Phys. Plasma 3, 2693 (1996).

${ }^{15}$ J. C. Adam, A. Héron, S. Guérin, G. Laval, P. Mora, and B. Quesnel, Phys. Rev. Lett. 78, 4765 (1997).

${ }^{16}$ J. Fuchs, J. C Adams, F. Amiranoff, S. D. Baton, P. Gallant, L. Gremillet, A. Héron, J. C. Kieffer, G. Laval, G. Malka, J. L. Miquel, P. Mora, H. Pepin, and C. Rousseaux, Phys. Rev. Lett. 80, 2326 (1998).

${ }^{17}$ M. Tabak, J. Hammer, M. E. Glinsky, W. L. Kruer, S. C. Wilks, J. Woodworth, E. M. Campbell, and M. D. Perry, Phys. Plasma 1, 1626 (1994).

${ }^{18}$ G. Li, R. Yan, C. Ren, T.-L. Wang, J. Tonge, and W. B. Mori, Phys. Rev. Lett 100, 125002 (2008).

${ }^{19}$ A. L. Lei, K. A. Tanaka, R. Kodama, K. Adumi, H. Habara, Y. Kitagawa, H. Kondo, T. Matsuoka, T. Tanimoto, T. Yabuuchi, K. Mima, K. Nagai, H. Nagatomo, T. Norimatsu, K. Sawai, K. Suzuki, W. Yu, H. Xu, X. Q. Yang, L. H. Cao, H. B. Cai, Y. Sentoku, A. Pukhov, R. Kumar, R. Snavely, R. Freeman, M. Yu, and J. Zheng, Phys. Plasma 16, 056307 (2009).

${ }^{20}$ T. Matsuoka, A. Lei, T. Yabuuchi, K. Adumi, J. Zheng, R. Kodama, K. Sawai, K. Suzuki, Y. Kitagawa, T. Norimatsu, K. Nagai, H. Nagatomo, Y. Izawa, K. Mima, Y. Sentoku, and K. A. Tanaka, Plasma Phys. Controlled Fusion, 50, 105011 (2008).

${ }^{21}$ M. Roth, T. E. Cowan, M. H. Key, S. P. Hatchett, C. Brown, W. Fountain, J. Johnson, D. M. Pennington, R. A. Snavely, S. C. Wilks, K. Yasuike, H. Ruhl, F. Pegoraro, S. V. Bulanov, E. M. Campbell, M. D. Perry, and H. Powell, Phys. Rev. Lett. 86, 436 (2001).

${ }^{22}$ V. Yu. Bychenkov, W. Rozmus, A. Maksimchuk, D. Umstadter, and C. E. Capjack, Plasma Phys. Rep. 27, 1017 (2001).

${ }^{23}$ S. Kar, A. P. L. Robinson, D. C. Carroll, O. Lundh, K. Markey, P. McKenna, P. Norreys, and M. Zepf, Phys. Rev. Lett. 102, 055001 (2009).

${ }^{24}$ G. S. Sarkisov, V. Yu. Bychenkov, V. T. Tikhonchuk, A. Maksimchuk, S. Y. Chen, R. Wagner, G. Mourou, and D. Umstadter, J. Exp. Theor. Phys. 66, 828 (1997). 
${ }^{25}$ K. Krushelnick, E. L. Clark, Z. Najmudin, M. Salvati, M. I. K. Santala, M. Tatarakis, A. E. Dangor, V. Malka, D. Neely, R. Allot, and C. Danson, Phys. Rev. Lett. 83, 737 (1999).

${ }^{26}$ S. P. Hatchett, C. G. Brown, T. E. Cowan, E. A. Henry, J. S. Johnson, M. H. Key, J. A. Koch, A. B. Langdon, B. F. Lasinski, R. W. Lee, A. J. Mackinnon, D. M. Pennington, M. D. Perry, T. W. Phillips, M. Roth, T. C. Sangster, M. S. Singh, R. A. Snavely, M. A. Stoyer, S. C. Wilks, and K. Yasuike, Phys. Plasmas 7, 2076 (2000).

${ }^{27}$ L. O. Silva, M. Marti, J. R. Davis, R. A. Fonseca, C. Ren, F. S. Tsung, and W. B. Mori, Phys. Rev. Lett. 92, 015002 (2004).

${ }^{28}$ T. Zh. Esirkepov, Y. Sentoku, K. Mima, K. Nishihara, F. Califano, F. Pegoraro, N. M. Naumova, S. V. Bulanov, Y. Ueshima, T. V. Liseikina, V. A. Vshivkov, and Y. Kato, J. Exp. Theor. Phys. 70, 82 (1999); Y. Fukuda, A. Ya. Faenov, M. Tampo, T. A. Pikuz, T. Nakamura, M. Kando, Y. Hayashi, A. Yogo, H. Sakaki, T. Kameshima, A. S. Pirozhkov, K. Ogura, M. Mori, T. Zh. Esirkepov, J. Koga, A. S. Boldarev, V. A. Gasilov, A. I. Magunov, T. Yamauchi, R. Kodama, P. R. Bolton, Y. Kato, T. Tajima, H. Daido, and S. V. Bulanov, Phys. Rev. Lett. 103, 165002 (2009).

${ }^{29}$ H. Chen, S. C. Wilks, J. D. Bonlie, E. P. Liang, J. Myatt, D. F. Price, D. D. Meyerhofer, and P. Beiersdofer, Phys. Rev. Lett. 102, 105001 (2009).

${ }^{30}$ P. Antici, J. Fuchs, E. d'Humières, J. Robiche, E. Brambrink, S. Atzeni, A. Schiavi, Y. Sentoku, P. Audebert, and H. Pépin, New J. Phys. 11, 023038 (2009).

${ }^{31}$ A. Henig, D. Kiefer, K. Markey, D. C. Gautier, K. A. Flippo, S. Letzring, R. P. Johnson, T. Shimada, L. Yin, B. J. Albright, K. J. Bowers, J. C. Fernández, S. G. Rykovanov, H.-C. Wu, M. Zepf, D. Jung, V. Kh. Liechenstein, J. Schreiber, D. Habs, and B. M. Hegelich, Phys. Rev. Lett. 103, 045002 (2009).

${ }^{32}$ C. Stoeckl, J. A. Delettrez, J. H. Kelly, T. J. Kessler, B. E. Kruschwitz, S. J. Loucks, R. L. McCrory, D. D. Meyerhofer, D. N. Maywar, S. F. B. Morse, J. Myatt, A. L. Rigatti, L. J. Waxer, J. D. Zuegel, and R. B. Stephens, Fusion Sci. Technol. 49, 367 (2006).

${ }^{33}$ J. Bromage. S.-W. Bahk, D. Irwin, J. Kwiatkowski, A. Pruyne, M. Millecchia, M. Moore, and J. D. Zuegel, Opt. Express, 16, 16561 (2008); B. Kruschwitz, S.-W. Bahk, J. Bromage, D. Irwin, and M. D. Moore, LLE Review Quarterly Report 124, LLE Document No. DOE/NA/28302-983 (2010).
${ }^{34}$ C. Dorrer, D. Irwin, A. Consentino, and J. Qiao, Conference on Lasers and Electro-Optics, OSA Technical Digest (CD) (Optical Society of America, 2010), paper JThE117 (2010).

${ }^{35}$ R. O. Fonseca, Lect. Notes Comput. Sci. 2331, 342 (2002).

${ }^{36}$ N. M. Naumova, J. Koga, K. Nakajima, T. Tajima, T. Zh. Esirkepov, S. V. Bulanov, and F. Pegoraro, Phys. Plasmas 8, 4149 (2001).

${ }^{37}$ N. M. Naumova, S. V. Bulanov, K. Nishihara, T. Zh. Esirkepov, and F. Pegoraro, Phys. Rev. E 65, 045402 (2002).

${ }^{38}$ S. V. Bulanov, T. Zh. Esirkepov, N. M. Naumova, F. Pegoraro, and V. A. Vshivkov, Phys. Rev. Lett. 82, 3440 (1999).

${ }^{39}$ D. Farina, and S. V. Bulanov, Phys. Rev. Lett. 86, 5289 (2001).

${ }^{40}$ N. M. Naumova, S. V. Bulanov, T. Zh. Esirkepov, D. Farina, K. Nishihara, F. Pegoraro, H. Ruhl, and A. S. Sakharov, Phys. Rev. Lett. 87, 185004 (2001).

${ }^{41}$ G. Sarri, D. K. Singh, J. R. Davies, F. Fiuza, K. L. Lancaster, E. L. Clark, S. Hassan, J. Jiang, N. Kageiwa, N. Lopes, A. Rehman, C. Russo, R. H. H. Scott, T. Tanimoto, Z. Najmudin, K. A. Tanaka, M. Tatarakis, M. Borghesi, and P. A. Norreys, Phys. Rev. Lett. 105, 175007 (2010).

${ }^{42}$ L. Romagnani, A. Bigongiari, S. Kar, S. V. Bulanov, C. A. Cecchetti, T. Zh. Esirkepov, M. Galimberti, R. Jung, T. V. Liseykina, A. Macchi, J. Osterholz, F. Pegoraro, O. Willi, and M. Borghesi, Phys. Rev. Lett. 105, 175002 (2010).

${ }^{43}$ A. Pukhov and J. Meyer-ter-Vehn, Phys. Plasmas 5, 1880 (1998).

${ }^{44}$ C. Gahn, G. D. Tsakiris, A. Pukhov, J. Meyer-ter-Vehn, G. Pretzler, P. Thirolf, D. Habs, and K. J. Witte, Phys. Rev. Lett. 83, 4772 (1999).

${ }^{45}$ J. Meyer-ter-Vehn and Z. M. Sheng, Phys. Plasmas 6, 641 (1999).

${ }^{46}$ A. Pukhov, Z.-M. Sheng, and J. Meyer-ter-Vehn, Phys. Plasmas 6, 2847 (1999).

${ }^{47}$ M. Tanimoto, S. Kato, E. Miura, N. Saito, K. Koyama, and J. K. Koga, Phys. Rev. E 68, 26401 (2003).

${ }^{48}$ S. P. D. Mangles, B. R. Walton, M. Tzoufras, Z. Najmudin, R. J. Clarke, A. E. Dangor, R. G. Evans, S. Fritzler, A. Gopal, C. Hernandez-Gomez, W. B. Mori, W. Rozmus, M. Tatarakis, A. G. R. Thomas, F. S. Tsung, M. S. Wei, and K. Krushelnick, Phys. Rev. Lett. 94, 245001 (2005).

${ }^{49}$ S. S. Bulanov, V. Yu. Bychenkov, V. Chvykov, G. Kalinchenko, D. W. Litzenberg, T. Matsuoka, A. G. R. Thomas, L. Willingale, V. Yanovsky, K. Krushelnick, and A. Maksimchuk, Phys. Plasmas 17, 043105 (2010). 